\title{
Article \\ Structural, Mechanical, and Dielectric Properties of Polydimethylsiloxane and Silicone Elastomer for the Fabrication of Clinical-Grade Kidney Phantom
}

\author{
Kamal Izdihar 1,2®), Hairil Rashmizal Abdul Razak 1,*(D), Nurzulaikha Supion 2, \\ Muhammad Khalis Abdul Karim ${ }^{3}{ }^{-}$, Nurul Huda Osman ${ }^{3}$ and Mazlan Norkhairunnisa ${ }^{4}$ \\ 1 Department of Radiology, Faculty of Medicine and Health Sciences, Universiti Putra Malaysia, \\ Seri Kembangan 43400, Selangor, Malaysia; izdihar.kamal@kpjuc.edu.my \\ 2 Department of Medical Imaging, School of Health Sciences, KPJ Healthcare University College, \\ Nilai 71800, N. Sembilan, Malaysia; zuezuzu1412@gmail.com \\ 3 Department of Physics, Faculty of Science, Universiti Putra Malaysia, \\ Seri Kembangan 43400, Selangor, Malaysia; mkhalis@upm.edu.my (M.K.A.K.); \\ nurulhuda@upm.edu.my (N.H.O.) \\ 4 Department of Aerospace Engineering, Faculty of Engineering, Universiti Putra Malaysia, \\ Seri Kembangan 43400, Selangor, Malaysia; norkhairunnisa@upm.edu.my \\ * Correspondence: rashmizal@upm.edu.my; Tel.: +60-16-437-5383
}

check for updates

Citation: Izdihar, K.; Abdul Razak, H.R.; Supion, N.; Karim, M.K.A.; Osman, N.H.; Norkhairunnisa, M. Structural, Mechanical, and Dielectric Properties of Polydimethylsiloxane and Silicone Elastomer for the Fabrication of Clinical-Grade Kidney Phantom. Appl. Sci. 2021, 11, 1172. https://doi.org/10.3390/app11031172

Received: 16 November 2020 Accepted: 22 December 2020 Published: 27 January 2021

Publisher's Note: MDPI stays neutral with regard to jurisdictional clai$\mathrm{ms}$ in published maps and institutional affiliations.

Copyright: (C) 2021 by the authors. Licensee MDPI, Basel, Switzerland. This article is an open access article distributed under the terms and conditions of the Creative Commons Attribution (CC BY) license (https:// creativecommons.org/licenses/by/ $4.0 /)$.

\begin{abstract}
This study aimed to introduce an alternative, inexpensive, and straightforward polymer with specific mechanical and dielectric properties suitable for the fabrication of a clinical-grade kidney phantom. Two polymer-based phantom materials, polydimethylsiloxane (PDMS) and silicone elastomer (SE), were investigated for their capability to meet the requirements. The concentration ratios of base to curing agent (B/C) were 9.5/1.5, 19/3, 10/1, 20/2, 10.5/0.5, and 21/1 for PDMS and $4.5 / 5.5,10 / 12,5 / 5,11 / 11,5.5 / 4.5$, and $12 / 10$ for SE. All samples were mixed, degassed, and poured into Petri dishes and small beakers. The polymer was cured under room temperature for $2 \mathrm{~h}$ and then demolded from the hard mold. The air bubbles produced were removed using a vacuum desiccator for $30 \mathrm{~min}$. All samples underwent mechanical testing (tensile strength and elastic modulus), and their dielectric properties were measured using a dielectric probe kit equipped with $85071 \mathrm{E}$ materials measurement software. The radiation attenuation properties were also measured using PhyX-Zetra for PDMS phantoms with the chemical formula $\mathrm{C}_{2} \mathrm{H}_{6} \mathrm{OSi}$. Small changes in base and cross-linker play an essential role in modifying the elastic modulus and tensile strength. The effective atomic number of PDMS showed a similar pattern with human kidney tissue at the intermediate energy level of $1.50 \times 10^{-1}$ to $1 \mathrm{MeV}$. Therefore, PDMS can potentially be used to mimic the human kidney in terms of tensile strength, flexibility, the acceptable real part of the complex dielectric constant $\varepsilon^{\prime} r$, and conductivity, which allows it to be used as a stable kidney phantom for medical imaging purposes.
\end{abstract}

Keywords: mechanical; dielectric; effective atomic number; PDMS; silicone elastomer; kidney phantom

\section{Introduction}

In medical imaging, calibration of the machine, operator training, surgical planning, and simulation are mostly conducted using a phantom. The therapeutic uses of radiation have proliferated and evolved since the discovery of $X$-rays. The occurrence of erythema and cell squamation have become apparent as harmful effects of high radiation doses. Therefore, people are reluctant to volunteer for radiation-based experiments. Physicists have developed a tissue substitute, known as a phantom, to simulate the response of real patients, which can be used for dosimetry measurements and identification of the limits of a particular system [1-4]. This phantom could be used to imitate living subjects and prevent unnecessary radiation delivered to human patients. 
A wide selection of materials have been studied to find an optimal outcome that best suits certain criteria for phantom development [5]. This has resulted in the fabrication of various materials, such as agar, silicone, polyvinyl alcohol (PVA), and polyacrylamide (PAA), to mimic human tissues [6]. The results have shown that agar is easy to prepare but does not have an excellent long-term stability compared to silicone, which can be considered as the material of choice for stable phantoms. A study comprehensively reviewed four different types of polymer-based materials for constructing medical phantoms: carrageenan-based polymer gel, polymer cross-linked aerogels, UV-curable silicone, and self-healing polymer materials [7]. The findings revealed that even though water-based silica and cellulose aerogel phantoms meet the imaging properties of human tissue, their volume may shrink and the fabrication is complex for larger-scale production. Nonetheless, a multimodal phantom has been successfully innovated with the use of polydimethylsiloxane (PDMS) and hydrophilic silicone [8,9]. The results demonstrated that the addition of hydrophilic silicone can improve the mechanical and imaging properties of a phantom. PDMS, or dimethicone, is a polymer that has been widely used for the fabrication and prototyping of microfluidic chips. The empirical formula of PDMS is $\left(\mathrm{C}_{2} \mathrm{H}_{6} \mathrm{OSi}\right) \mathrm{n}$ and its fragmented formula is $\mathrm{CH}_{3}\left[\mathrm{Si}\left(\mathrm{CH}_{3}\right) 2 \mathrm{O}\right] \mathrm{nSi}\left(\mathrm{CH}_{3}\right) 3$, where $n$ represents the number of monomer repetitions.

PDMS has an elastic modulus of $360-870 \mathrm{kPa}$, which is larger than the elastic modulus of a real kidney, with $180.32 \pm 11.11 \mathrm{kPa}$ (mean $\pm \mathrm{SD}$ ) and $95.64 \pm 9.39 \mathrm{kPa}$ under axial and transversal loadings, respectively [8]. Elastomers are required to undergo vulcanization, which is the formation of a cross-link between long polymer chains. This feature controls the ability of the elastomer to reconfigure its structure when stress is applied, indicating the elasticity. The covalent cross-linkages ensure that the elastomer returns to its original configuration when the stress is removed and result in permanent deformation. Cross-links can be introduced into polysiloxanes by a range of methods, including (i) the use of a tri- or tetrafunctional siloxane comonomer during polymerization; (ii) the incorporation of a thermal initiator, which attracts hydrogen atoms from PDMS, thus resulting in cross-linking; or (iii) the exposure of PDMS to high-energy irradiation. Figure 1 shows the method of PDMS cross-linking proposed by a previous author [9]. Cross-linking reactions can also be applied with the intention to improve the surface of a cured PDMS sample using hyperthermal hydrogen-induced cross-linking (HHIC) [10].
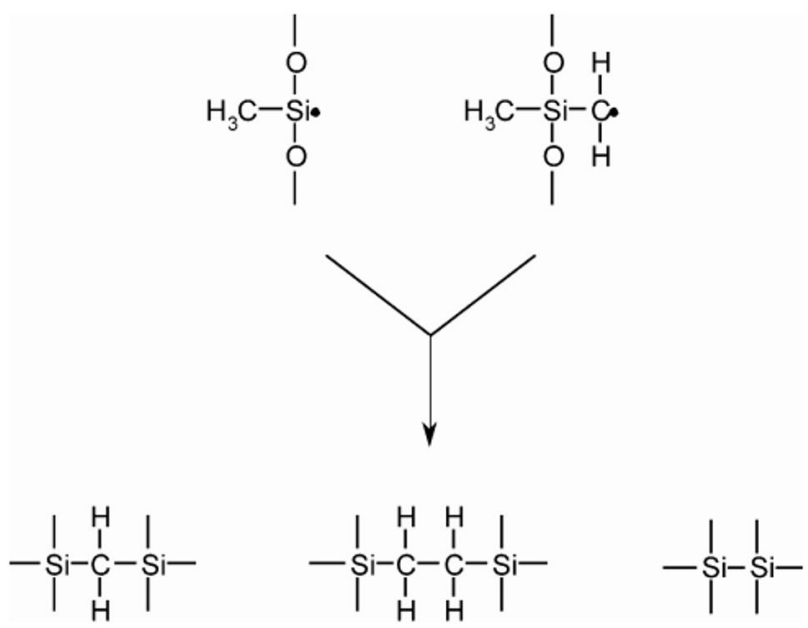

Figure 1. Cross-linking of polydimethylsiloxane (PDMS).

In this paper, we also discuss the group of polymers known as silicone elastomers (SEs). SE is typically cross-linked using hydrosilylation (usually by Pt catalysis), condensation (usually by Sn catalysis), and radical reaction [11]. In Figure 2, the two most commonly used curing chemistries are illustrated. SE is widely used due to its favorable properties, namely flexibility and durable dielectric insulation, and its exceptional barrier properties 
against environmental contaminants. SE is superior to PDMS in terms of simpler fabrication (it can be cured at room temperature without the use of an oven) because the premixes have low viscosity. The amount of curing agent required is robust and not as sensitive to PDMS formulations. In addition, SE provides more flexible and stretchable capabilities. Therefore, it would be beneficial for researchers to compare the effectiveness of PDMS with that of SE.

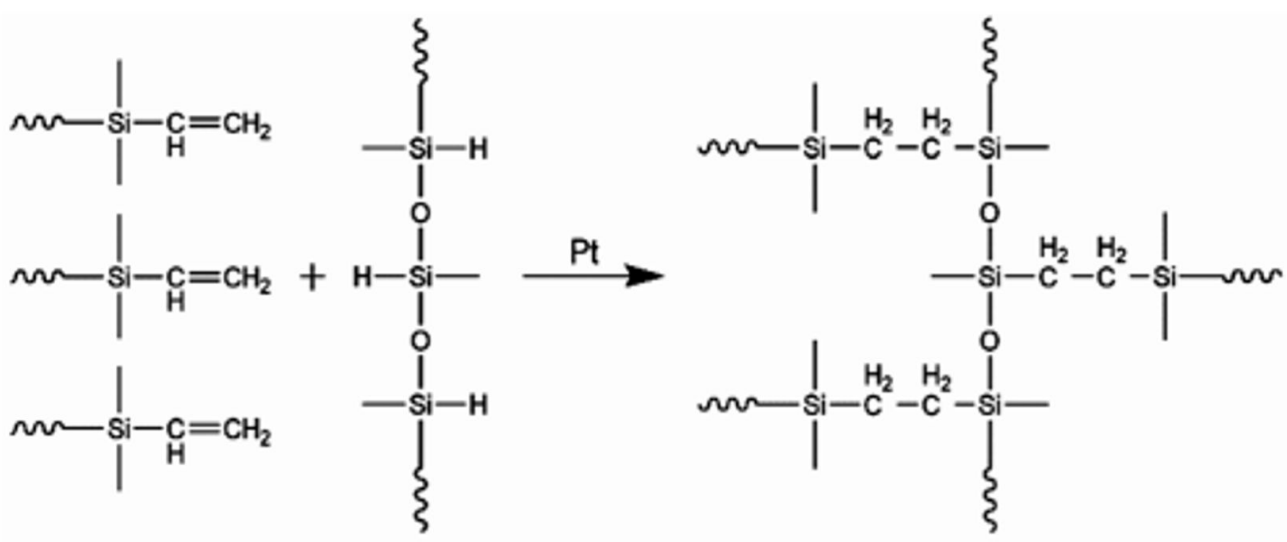

Figure 2. Cross-linking chemistries for silicone elastomer by hydrosilylation reactions.

As the amount of cross-linker may affect the strength and stability of samples, the samples used in this study were prepared with minimal differences. To the best of our knowledge, studies on the small ratio are still limited, so we investigated the effects of small differences in the curing agent in the cross-linking process in both PDMS and SE. The PDMS network's cross-linking with base to curing agent mass ratios of 5:1, 10:1, 16.7:1, 25:1, and $33: 1$ had an elastic modulus ranging from 0.57 to $3.7 \mathrm{MPa}$ and was linearly proportional to the amount of cross-linker [12]. A kidney phantom composed of siloxane (silicone) is the most widely used polymer for mimicking physical, optical, and thermal properties $[13,14]$.

Adams et al. successfully fabricated a kidney phantom using silicone elastomer, PDMS, and agarose, and, at the same time, constructed a collecting system and outer mold from 3D-printed models [15]. They proved that a combination of tissue-mimicking material and 3D-printed models can further improve the performance of the phantom for various imaging modalities such as CT scan, ultrasound, and endoscopy. In this study, PDMS and SE were prepared using the standard cross-linking formula by the manufacturer, and mechanical testing was performed. PDMS and SE with different thicknesses were fabricated using different cross-linking methods. Additionally, the dielectric and mean attenuation properties of the PDMS material were quantified.

The aim of this study was to formulate a tissue-mimicking material for kidney phantoms because the possibility of underestimated lesions is very high in the kidney, and the radiation dose to the kidney is a primary concern. Apart from that, it is important to distinguish between PDMS and SE because each material has superior flexibility depending on the amount of cross-linker. As rigidity is controlled by cross-linker density, the threshold limit is a vital factor for observing the performance of that material. To the best of our knowledge, most of the phantoms are not tested for material sustainability and effective atomic number $\left(Z_{\text {eff }}\right)$, instead only focusing on their imaging properties. Therefore, we focused on polymer-based phantoms with a specific mechanical property and effective atomic number to determine the most suitable material for kidney phantoms.

\section{Materials and Methods}

\subsection{Polydimethylsiloxane (PDMS) and Silicone Elastomer (SE) Fabrication}

Two different silicones were selected based on the differences in their viscosity, dielectric current, and mechanical properties for fabricating the phantom. The silicone elastomer Ecoflex 00-20 (viscosity of 3000 cps and translucent color) was supplied by Smooth-on (PA, 
United States), and PDMS Sylgard 184 (density $0.965 \mathrm{~g} / \mathrm{cm}^{3}$, viscosity (mixed) $3500 \mathrm{cps,}$ and high transparency) was supplied by Dow Corning (MI, United States).

We formulated different PDMS solutions to investigate possible alterations in their properties due to deviation from the recommended 10/1 weight ratio [16]. The standard mixing ratio for PDMS was ten parts of base and one part of curing agent (dimethyl vinylated and trimethylated silica, respectively) [8]. The pre-polymer PDMS and curing agent were combined at various weight ratios, designated as 9.5/1.5, 10/1, and 10.5/0.5. Initially, two parts of PDMS were weighed and stirred in a beaker using a hand stir for five minutes, then the solution was placed in a vacuum desiccator for $30 \mathrm{~min}$ to remove the air bubbles. Before the PDMS was casted, a passivation layer (Ease release 200) was applied on the Petri dish to avoid strong adhesion between PDMS and Petri dish so that it could be easily peeled off. The mixtures were then heated in the oven at $80^{\circ} \mathrm{C}$ for $30 \mathrm{~min}$ and the temperature was increased to $100^{\circ} \mathrm{C}$ for another hour. The cured sample was left to cool at room temperature.

SE fabrication consisted of two parts of mixture, in which part A (vinyl silicone, white carbon black, and organic platinum catalyst) was the base and part B (vinyl silicone and white carbon black) contained the curing agent [11]. These two parts were combined at different weight ratios, at base to curing agent ratios of 4.5/5.5, 5/5, and 5.5/4.5 for the thin (weight of $10 \mathrm{~g}$ ) and thick (weight of $20 \mathrm{~g}$ ) samples, as tabulated in Table 1. Part A and part $\mathrm{B}$ were stirred thoroughly before dispensing. A release agent, Ease Release $\mathrm{C}^{\mathrm{TM}}$ 200, was sprayed to prevent mold degradation. Then, the sample was poured from the mixing container into the Petri dish. Vacuum degassing was used to eliminate trapped air. We allowed the rubber to cure as prescribed at room temperature $\left(73^{\circ} \mathrm{F} / 23^{\circ} \mathrm{C}\right)$ before demolding.

Table 1. Samples with various mixtures of PDMS and Silicone Elastomer (SE).

\begin{tabular}{ccccc}
\hline \multirow{2}{*}{ No. } & \multicolumn{2}{c}{ PDMS } & \multicolumn{2}{c}{ SE } \\
\cline { 2 - 5 } & $\begin{array}{c}\text { Sample } \\
\text { (Thin, 11 g) }\end{array}$ & $\begin{array}{c}\text { Sample } \\
\text { (Thick, 22 g) }\end{array}$ & $\begin{array}{c}\text { Sample } \\
\text { (Thin, 10 g) }\end{array}$ & $\begin{array}{c}\text { Sample } \\
\text { (Thick, 22 g) }\end{array}$ \\
\hline 1 & PDMS 9.5/1.5 & PDMS 19/3 & SE 4.5/5.5 & SE 10/12 \\
\hline 2 & PDMS 10/1 & PDMS 20/2 & SE 5/5 & SE 11/11 \\
\hline 3 & PDMS 10.5/0.5 & PDMS 21/1 & SE 5.5/4.5 & SE 12/10 \\
\hline
\end{tabular}

\subsection{Material Characterizations}

\subsubsection{Tensile Strength}

Tensile tests were conducted to determine the strength and behavior of PDMS and SE under tension. The tensile strengths of the PDMS and SE were measured using the Instron 4411 electromechanical material testing system (Instron Corporation, Canton, MA, USA). The samples were cut to a dumbbell-shape test. Figure 3 shows a schematic illustration of the sample geometry prior to testing. The materials were pulled by an applied force of less than $15 \mathrm{~N}$ [7]. The load and crosshead velocity of $254.00 \mathrm{~mm} / \mathrm{min}$ were controlled and digitally acquired at $200 \mathrm{~Hz}$ using Merlin software V.22043 (Instron Corporation). From the tensile analysis, the modulus of elasticity (MOE) can be calculated using the following formula [8]:

$$
E=\frac{\sigma}{\varepsilon}
$$

where $E$ is the slope of the line in this region, and stress $(\sigma)$ is proportional to strain $(\varepsilon)$. 


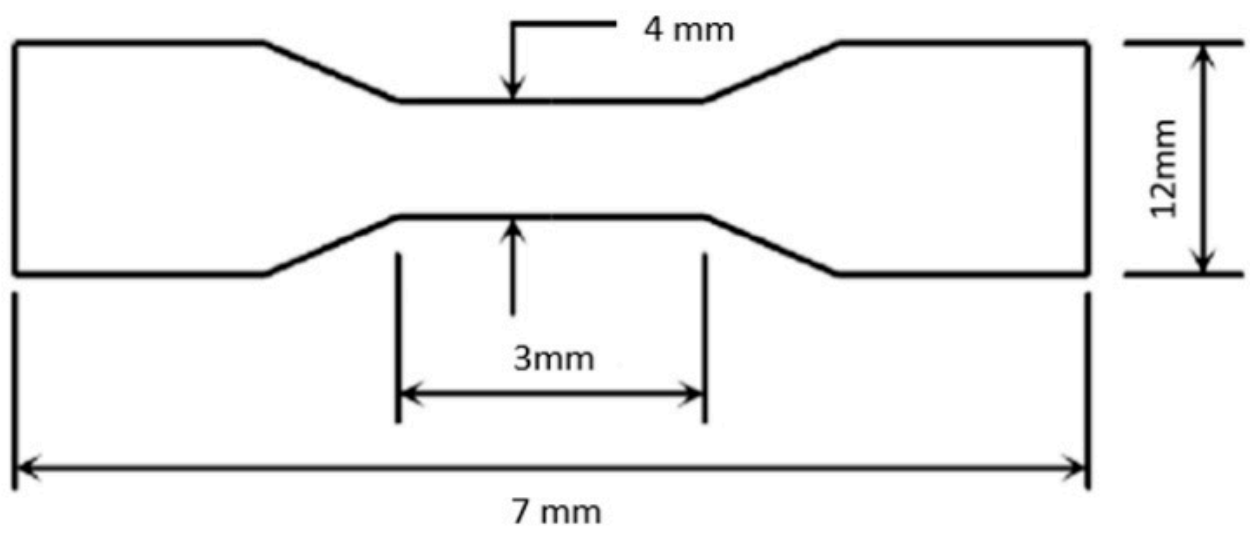

Figure 3. A schematic illustration of the sample geometry prior to testing.

\subsubsection{Dielectric Testing}

The open-ended coaxial method was used to find the electromagnetic parameters of the polymers. The tested sample diameter was $25 \mathrm{~mm}$, while the thickness was kept at approximately $25 \mathrm{~mm}$, and the sample was measured by touching it to the flat face of a solid material that operating within the frequency range of $1.39-19.22 \mathrm{GHz}$ at room temperature. The measurement system comprised an open-ended coaxial probe (85071E, Keysight, CA, USA) connected to a vector network analyzer. The samples were cured in a small beaker using the same method, as shown in Figure 4a. The cured samples are shown in Figure $4 \mathrm{~b}$. The vector network analyzer consisted of a signal source, a receiver, and a display, as shown in Figure 5a,b. The source launches a signal at a single frequency to the material under test. The receiver is tuned to detect the reflected frequency and transmitted signals from the material. The measured response produces the magnitude and phase data at that frequency.
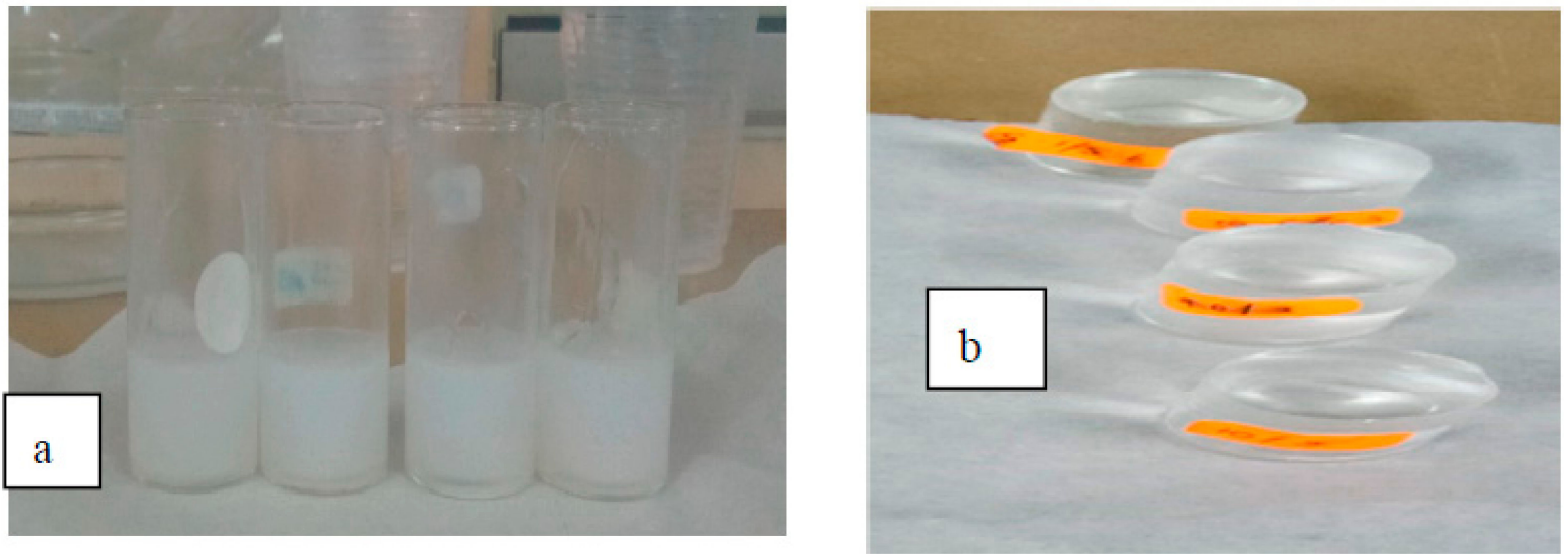

Figure 4. (a) Four cured SE samples in small beakers. (b) Four cured PDMS samples being removed from the glass tube. 


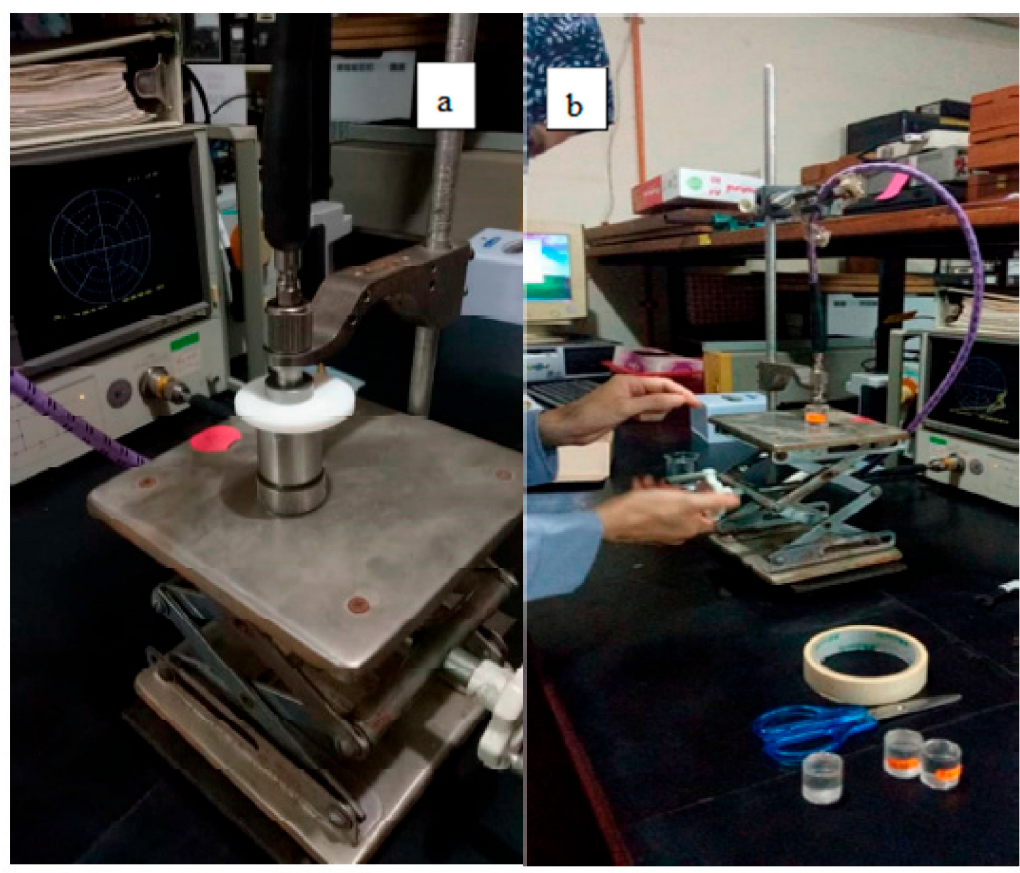

Figure 5. (a) Calibration standard of the probe is set by using a standard sample. (b) PDMS.

The connected dielectric measurement instrument predictably applies the electromagnetic fields before the measurement process. The measured data are then converted into permittivity or permeability values. The real part of the complex dielectric constant $\varepsilon^{\prime} r(K)$ is equivalent to the relative permittivity $(\varepsilon r)$, or the absolute permittivity $(\varepsilon)$ relative to the permittivity of free space $\left(\varepsilon_{0}\right)$. The real part of permittivity $\left(\varepsilon^{\prime} r\right)$ is the dielectric constant, and the imaginary part is the dielectric loss $\varepsilon r^{\prime \prime} . \varepsilon^{\prime} r$ is a measure of how much energy from an external electric field is stored in the material. The imaginary part of permittivity ( $\left.\varepsilon r^{\prime \prime}\right)$ is called the loss factor, which is a measure of how dissipative or lossy the material is to an external electric field.

$$
K=\frac{\varepsilon}{\varepsilon_{0}}=\varepsilon r=\varepsilon^{\prime} r-j \varepsilon r^{\prime \prime}
$$

The conductivity is measured by considering the imaginary part of permittivity $\varepsilon r^{\prime \prime}$, which represents the mechanisms of electromagnetic losses inside the material due to molecular polarization and electron movement. Hence, the conductivity, $\sigma$, can be computed as follows [17]:

$$
\sigma(f)=2 \pi f \varepsilon r^{\prime \prime}(f) \varepsilon_{0}
$$

2.2.3. Radiation Attenuation Properties: Mean Attenuation Properties and Effective Atomic Number $\left(Z_{\text {eff }}\right)$

Phy-X/ZeXTRa is free online software developed with an easy-to-use graphic user interface (GUI) interface and used to calculate the $Z_{\text {eff }}$ of photons (both photon interactions and photon energy absorption) in the energy region of $1 \mathrm{keV}-20 \mathrm{MeV}$ and of electrons, protons, alpha particles, and ions in the energy region of $1 \mathrm{MeV}-1 \mathrm{GeV}$. This software can calculate the $Z_{\text {eff }}$ of more than 180 predefined materials of interest in the field of medical physics. The total weight number and fractions of the mole inserted shall be equal to 1 (or 100\%).

\section{Results}

\subsection{Tensile Strength}

The results of the tensile tests varied with different mixtures of base and curing agent. Figures 6 and 7 show that the standard mixing ratio was consistently high for both the PDMS Young's modulus and tensile strength. The tensile strength increased with increasing amount of cross-linker. However, further increases in the amount of cross-linker decreased 
the tensile strength by reducing the amount of curing agent. PDMS 10.5/0.5 and PDMS $21 / 1$ reduced the stiffness and flexibility of the samples. The PDMS samples were observed to have lower tensile and Young's modulus values with the increasing weight and thickness of the specimens of PDMS 19/3, PDMS 20/2, and PDMS 21/1. This trend showed that lighter samples are consistently stiffer than heavier samples.

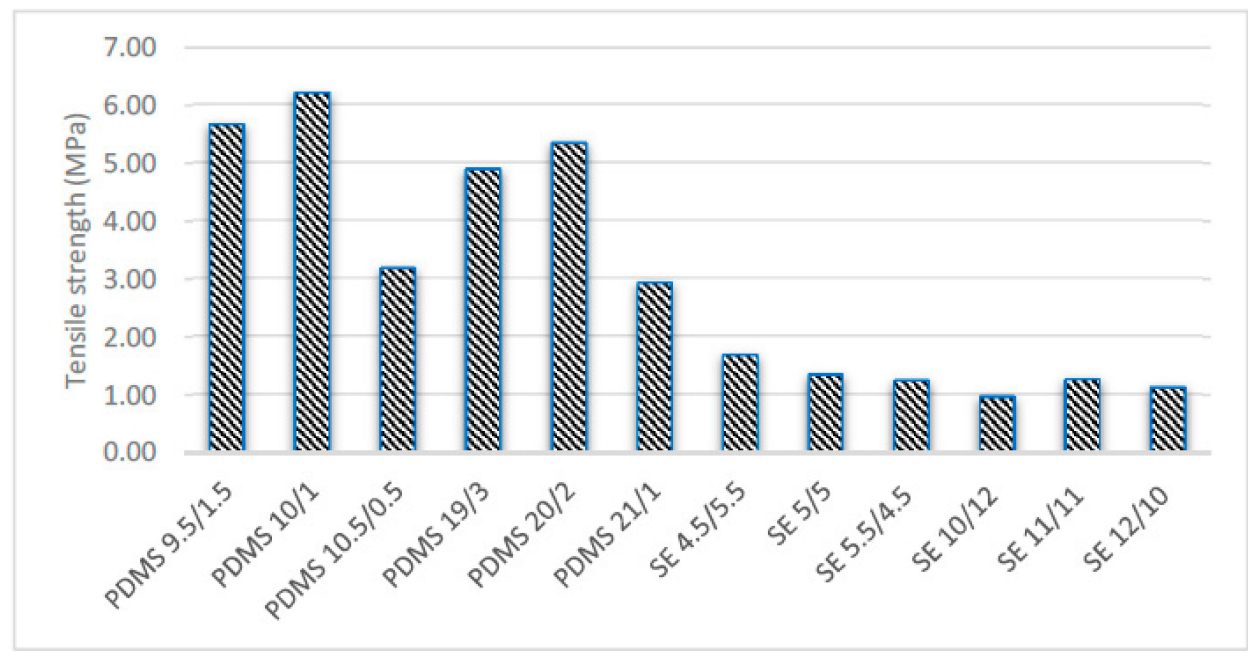

Figure 6. Tensile strength for various mixtures and thicknesses.

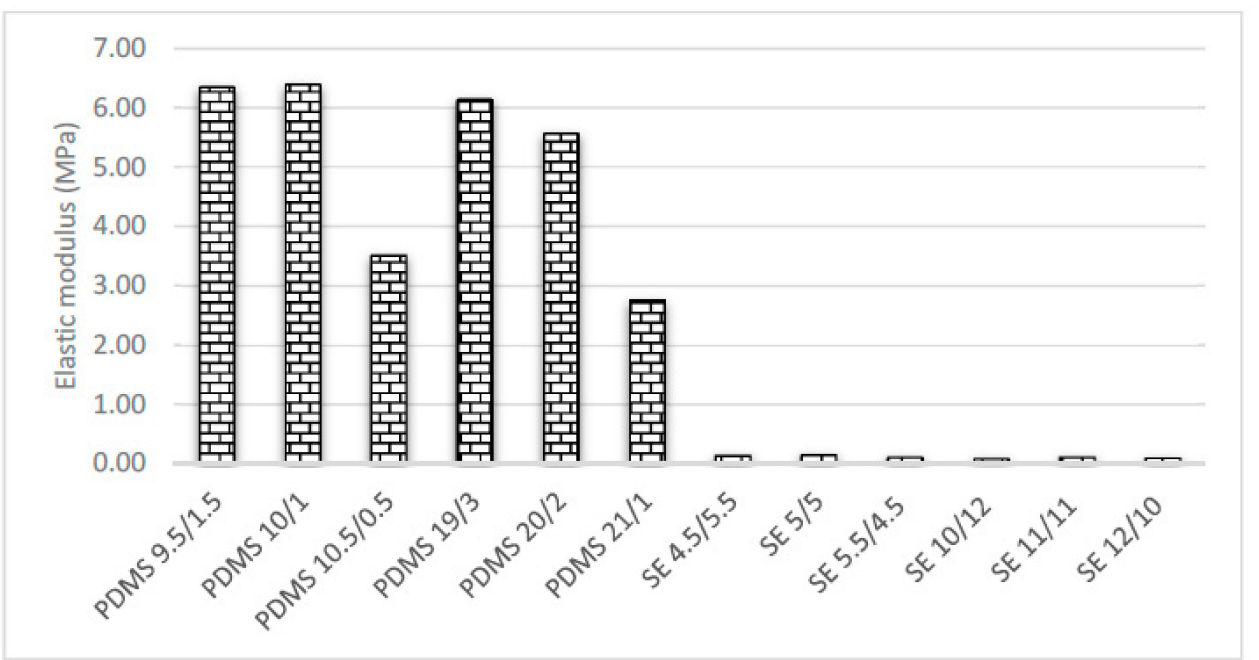

Figure 7. Elastic modulus for various mixtures and thicknesses.

On the other hand, SE did not result in conclusive findings since the graph was not consistent for either Young's modulus or tensile strength (Figures 6 and 7). The Young's modulus of our SE samples was relatively higher, while the tensile strength was in good agreement with that reported in previous studies [15]. However, the thin samples followed the same graph pattern as PDMS. The standard mixing ratio of SE $5 / 5$ was found to be optimum as the elastic modulus was higher compared to those of the other mixing ratios.

\subsection{Dielectric Measurement}

Based on Equation (2), the real part of the complex dielectric constant $\varepsilon^{\prime} r$ and loss are shown in Figure 8a,b. The dielectric constant $\varepsilon^{\prime} r$ obtained from this study showed a similar pattern for both SE and PDMS samples. The dielectric constant $\varepsilon^{\prime} r$ for SE was higher than that of the PDMS samples with a similar range, between 12 and 48 . The maximum difference in the dielectric constant $\varepsilon^{\prime} r$ for SE 5.5/4.5 was higher than that in PDMS 10.5/0.5 
by a factor of \pm 1.2 . Figure 8 a shows a significant drop in the dielectric constant, which corresponds to the maximum frequency for the dielectric loss shown in Figure 8b. This transition represents the frequency at which the dipole polarization is completely out of phase with the applied oscillating electric field. The relative permittivity decreases at high frequency as $\gamma, \beta$, and $\alpha$ dispersion occurs. In the gigahertz region, $\gamma$ dispersion occurs due to water molecule polarization, and in the kilohertz region, $\beta$ dispersion occurs due to the polarization of cellular membranes, whereas at low frequency, $\alpha$ dispersion is associated with the ionic diffusion of cellular membranes. Since both PDMS 10/1 and SE 5/5 samples had a high Young's modulus and tensile strength, we illustrate the dielectric constant $\varepsilon^{\prime} r$ and conductivity of standard PDMS $10 / 1$ and SE 5/5 samples for a frequency between 0.2 and 19.2 GHz in Figure 9.
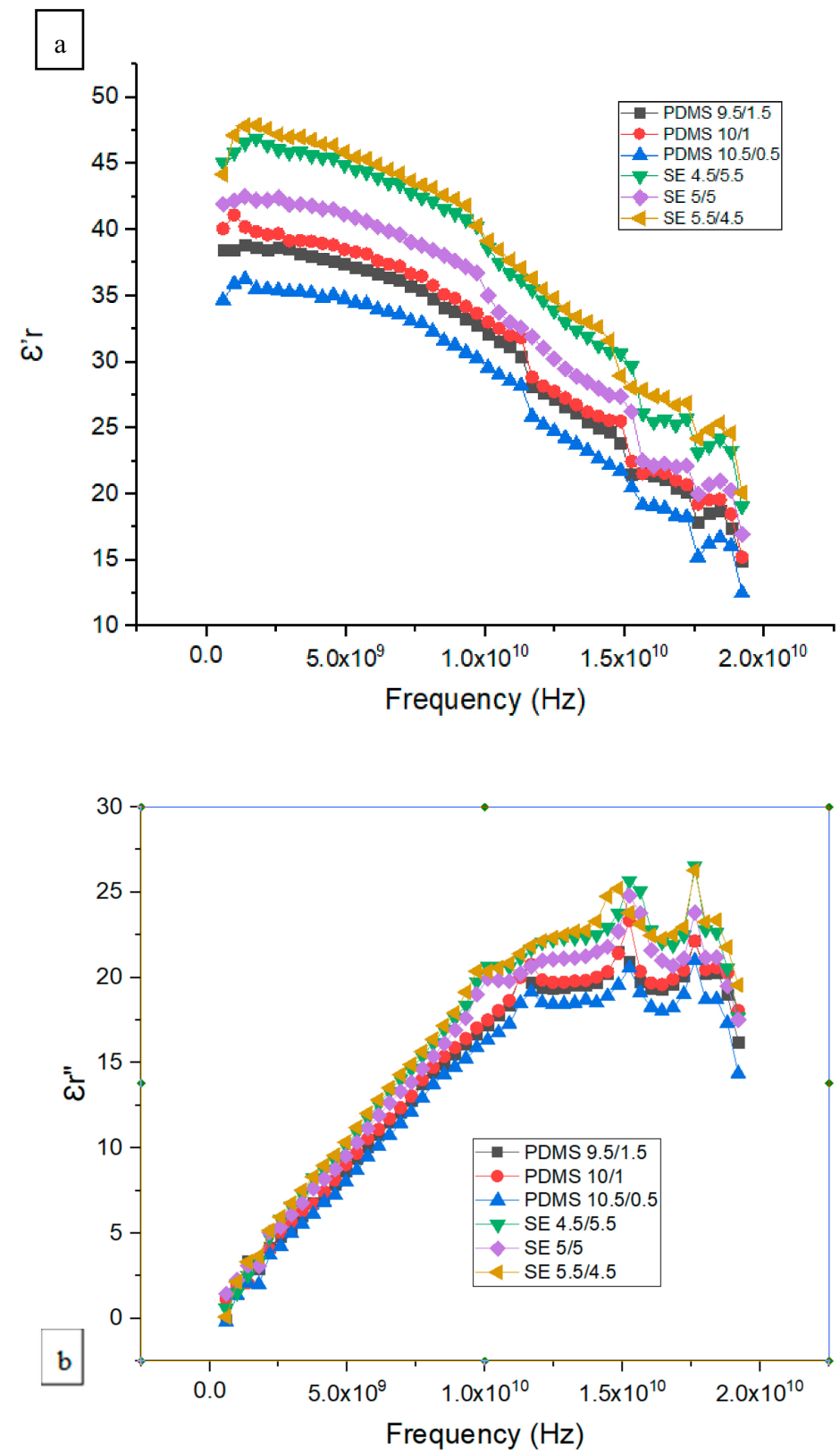

Figure 8. (a) The real part of the complex dielectric constant $\varepsilon^{\prime} r$ and (b) the dielectric loss $\varepsilon r^{\prime \prime}$ for various mixtures of PDMS and SE. 


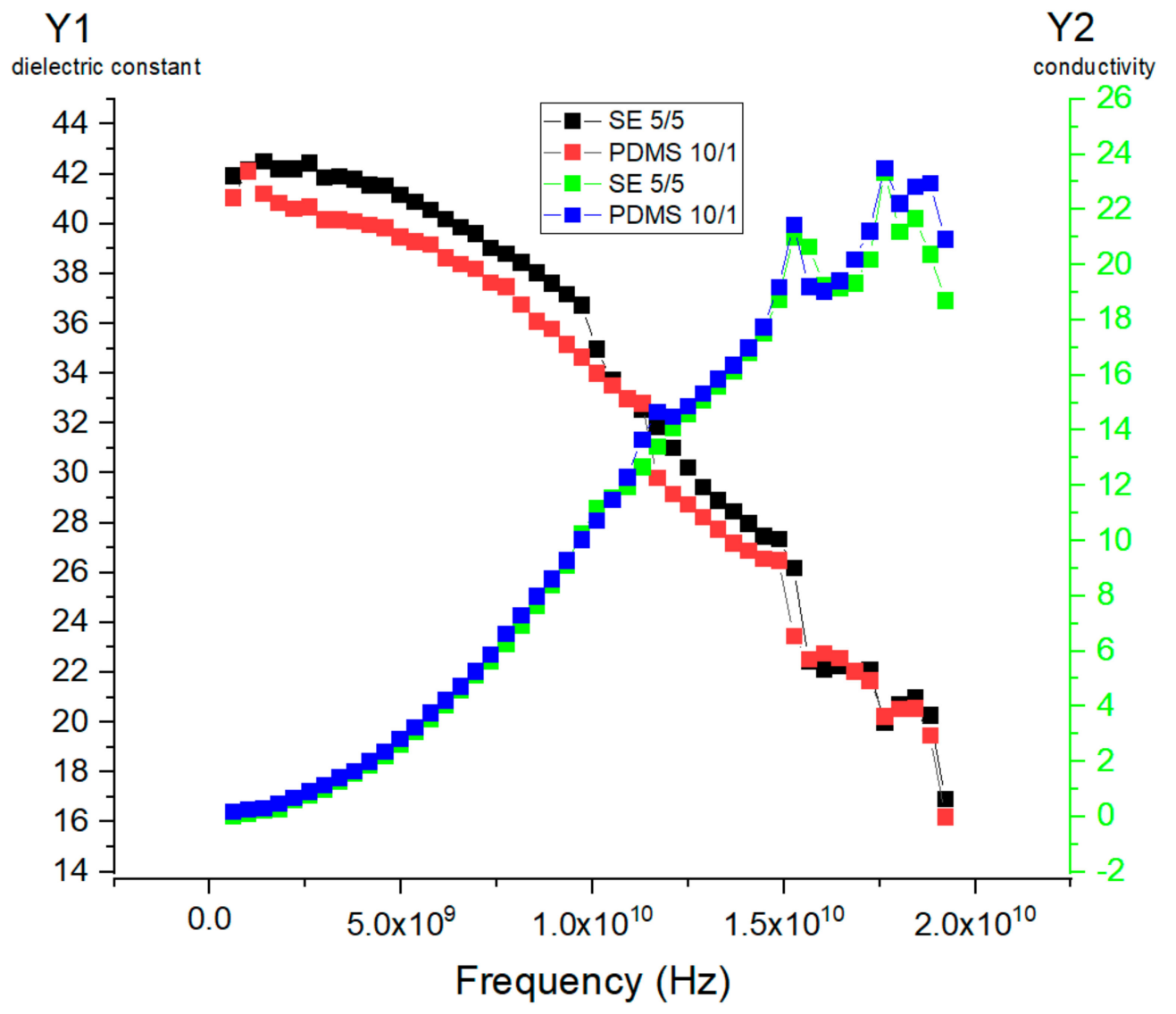

Figure 9. (Y1) The real part of the complex dielectric constant $\varepsilon^{\prime} r$ of standard PDMS and SE 5/5. (Y2) The conductivity s/m $(\sigma)$ of standard PDMS 10/1 and SE 5/5.

3.3. Radiation Attenuation Properties: Mass Attenuation Coefficient, and Effective Atomic Number

Based on our earlier analyses, standard PDMS 10/1 is thought to be the optimum level. Therefore, further evaluations of radiation attenuation properties were conducted. The mass attenuation coefficient and effective atomic number of standard PDMS 10/1 are tabulated in Table 2. The effective atomic number is a photon interaction parameter, which is used for dosimetric properties. The studied silicone samples have relatively moderate $\mathrm{Z}$ elements. Therefore, there is a need to determine the attenuation properties and effective atomic number of these samples. Our PDMS samples had $Z_{\text {eff }}$ values similar to those reported in an earlier study by Majunatha [18]. 
Table 2. Radiation attenuation properties of PDMS 10/1.

\begin{tabular}{|c|c|c|}
\hline $\begin{array}{l}\text { Energy } \\
\text { MeV }\end{array}$ & $\begin{array}{c}\text { Mass Attenuation } \\
\text { Coefficient (MAC) } \\
\mathrm{cm}^{2} / \mathrm{g}\end{array}$ & $\begin{array}{l}\text { Effective Atomic Number } \\
\left(Z_{\text {eff }}\right)\end{array}$ \\
\hline $1.50 \times 10^{-2}$ & 4.603 & 11.41 \\
\hline $2.00 \times 10^{-2}$ & 2.050 & 10.35 \\
\hline $3.00 \times 10^{-2}$ & 0.738 & 8.02 \\
\hline $4.00 \times 10^{-2}$ & 0.417 & 6.38 \\
\hline $5.00 \times 10^{-2}$ & 0.300 & 5.45 \\
\hline $6.00 \times 10^{-2}$ & 0.246 & 4.93 \\
\hline $8.00 \times 10^{-2}$ & 0.198 & 4.45 \\
\hline $1.00 \times 10^{-1}$ & 0.176 & 4.26 \\
\hline $1.50 \times 10^{-1}$ & 0.149 & 4.10 \\
\hline $2.00 \times 10^{-1}$ & 0.135 & 4.05 \\
\hline $3.00 \times 10^{-1}$ & 0.116 & 4.02 \\
\hline $4.00 \times 10^{-1}$ & 0.103 & 4.01 \\
\hline $5.00 \times 10^{-1}$ & 0.094 & 4.01 \\
\hline $6.00 \times 10^{-1}$ & 0.087 & 4.01 \\
\hline $8.00 \times 10^{-1}$ & 0.076 & 4.00 \\
\hline $1.00 \times 10$ & 0.069 & 4.00 \\
\hline $1.50 \times 10$ & 0.056 & 4.01 \\
\hline $2.00 \times 10$ & 0.048 & 4.02 \\
\hline $3.00 \times 10$ & 0.039 & 4.08 \\
\hline $4.00 \times 10$ & 0.034 & 4.15 \\
\hline $5.00 \times 10$ & 0.030 & 4.22 \\
\hline $6.00 \times 10$ & 0.028 & 4.30 \\
\hline $8.00 \times 10$ & 0.025 & 4.46 \\
\hline $1.00 \times 10^{1}$ & 0.023 & 4.62 \\
\hline $1.50 \times 10^{1}$ & 0.021 & 4.97 \\
\hline
\end{tabular}

\section{Discussion}

With regard to the tensile test results, PDMS and SE show different performances at different ratios of base to curing agent. The suggested ratios are 10/1 for PDMS and $5 / 5$ for SE. The elastic modulus increased dramatically with decreasing thickness. This finding is consistent with a previous study [19]. The phenomena of stretching, breaking, and deformation are critical in that the arrangement of polymer in PDMS controls its mechanical properties. The SE 10/12 ratio was consistently lower compared with the other SE samples due to lower base-to-catalyst ratio and thicker sample. Therefore, part A (base-to-catalyst ratio) was found to be a fundamental property for the strength of a sample. Overall, as observed in the SE graph, the lower the amount of part A (catalyst-to-base ratio), the more likely it becomes unstable. Nevertheless, there is a slight difference concerning the impact of the curing agent on the material properties. This finding is consistent with earlier studies in which the sample was mixed with higher amounts of curing agent $[12,20]$. In addition, a higher amount of base reduces the strength of the overall compound properties due to the lack of interaction between the base and the curing agent [20].

According to Zhixin (Tampa, Florida), increasing the amount of cross-linker by up to $20 \%$ could stiffen the PDMS network [12]. However, other reports showed that for 
higher concentrations of curing agent, the modulus is reduced since the cross-link sites are saturated, and excess curing agent leads to a void or dilution of the network. This reduces the stiffness, especially when measured in tension, which is inconsistent with the results of the present study, where a higher amount of curing agent reduced the elastic modulus of PDMS and SE. The density of PDMS was $0.965 \mathrm{~g} / \mathrm{cm}^{3}$, which is similar to the mass density of the kidney, $1.066 \mathrm{~g} / \mathrm{cm}^{3}$. The flexibility and stable mechanical properties of PDMS are beneficial for phantom storage and transportation.

There is an apparent difference between the PDMS and SE samples. The dielectric result can be varied with different amounts of base and curing agents. In this study, the dielectric constant $\varepsilon^{\prime} r$ was lower than that of water but higher than the dielectric constant $\varepsilon^{\prime} r$ of other polymers [21]. Our thick samples might not dry fully, and this will dramatically alter the desired dielectric properties [21]. The conductivity value found in this study is comparable with those reported in other literature, ranging from 5 to $10 \mathrm{~S} / \mathrm{m}$ [5]. Muscles and high-density tissues have relatively high conductivity and permittivity, while adipose tissue has comparatively low permittivity and conductivity values [5]. Additionally, silicone possesses favorable dielectric properties such as high dielectric breakdown strength and high resistivity [11]. For most dielectric materials, ionic conductivity plays a significant role at frequencies less than $200 \mathrm{MHz}$. Basically, insulators have a lower dielectric constant $\varepsilon^{\prime} r$; thus, in this study, PDMS was a better insulator compared to SE.

The attenuation properties of our standard phantom were studied to ensure that it could mimic human tissue. Therefore, Phy-X/ZeXTRa software was used to calculate $Z_{\text {eff }}$ [22]. The effective atomic number is constant in the intermediate-energy region, whereas noticeable variation was observed for low- $(<20 \mathrm{keV})$ as well as high-energy regions [23]. Overall, the effective atomic number of PDMS is around 4.00 to 5.00 in a higher-energy range $\left(\geq 1.00 \times 10^{-1} \mathrm{MeV}\right)$ and comparable with the effective atomic number of the kidney found in literature [18]. This $Z_{\text {eff }}$ is lower at the intermediate energy range due to the dominance of pair production and scattering effects. This study only focused on the chemical formula of PDMS since PDMS is found to be the material of choice to be used as the kidney phantom due to its effective atomic number, high elastic modulus, and comparable real part of the complex dielectric constant $\varepsilon^{\prime} r$, as reported by other researchers $[4,17]$.

Overall, we proved that a minimal difference in curing agent not exceeding $20 \%$ (curing agent to base ratio) between SE samples in this study does not alter the elastic modulus substantially. In contrast, PDMS is more sensitive to the amount of cross-linker compared to SE; the amount of cross-linker varies by $5 \%, 10 \%$, and $16 \%$, yet the elastic modulus changes substantially. Hence, the $5 \%$ cross-linker reduces the elastic modulus by half and deteriorates the strength of the sample regardless of thickness. The dielectric properties can also be affected by the amount of cross-linker. As the level of curing increases, the maximum dielectric loss shifts toward lower frequency, while the change in the dielectric constant at $\alpha$ static with $\alpha$ infinity diminishes [21]. This behavior represents the gradual transition from the rubbery to the glassy state of the polymer with an increase in cross-link density.

\section{Conclusions}

In conclusion, this research work presents a simple method of characterizing a sample by investigating the mechanical and dielectric properties of various mixtures of the polymers PDMS and SE. From an effective atomic number perspective, PDMS showed a pattern similar to kidney tissue at intermediate energy level. Thus, it is recommended as the material of choice for kidney phantoms. Additionally, small changes in the amount of cross-linker could alter the elastic modulus, and more caution should be exercised when reducing the cross-linker from its standard value as it deteriorates the sample's stability. It is compulsory to modify the cross-linking at a range of $\pm 20 \%$, and addition of water and hydrogen silicone could improve the mechanical properties of the phantom. Moreover, its transparency has made the process of making texture phantoms easier. To conclude, 
PDMS could mimic human kidney tissue in terms of flexibility, an acceptable real part of the complex dielectric constant $\varepsilon^{\prime} r$, and conductivity, demonstrating its suitability as the material of choice to be used as a stable kidney phantom in the field of medical imaging.

Author Contributions: Data curation, M.K.A.K.; Formal analysis, M.K.A.K.; Funding acquisition, H.R.A.R.; Investigation, K.I., N.S. and N.H.O.; Project administration, K.I.; Resources, M.K.A.K.; Writing-review \& editing, K.I., M.K.A.K., M.N. All authors have read and agreed to the published version of the manuscript.

Funding: Geran Putra of Universiti Putra Malaysia with the project number GP/IPS/UPM/9683700.

Institutional Review Board Statement: The study did not require ethical approval. Ethical review and approval were waived for this study, due to this study not involving humans or animals.

Informed Consent Statement: Not applicable.

Acknowledgments: The authors wish to acknowledge support from Geran Putra of Universiti Putra Malaysia with the project number GP/IPS/UPM/9683700.

Conflicts of Interest: The authors declare no conflict of interest.

\section{References}

1. Varghese, B.; Hwang, D.; Cen, S.Y.; Levy, J.; Liu, D.; Lau, C.; Rivas, M.; Desai, B.; Goodenough, D.J.; Duddalwar, V.A. Reliability of CT-based texture features: Phantom study. J. Appl. Clin. Med. Phys. 2019, 20, 155-163. [CrossRef] [PubMed]

2. Nikawa, Y.; Chino, M.; Kikuchi, K. Soft and dry phantom modeling material using silicone rubber with carbon fiber. IEEE Trans. Microw. Theory Tech. 1996, 44, 1949-1953. [CrossRef]

3. Murat, H.; Karim, M.; Harun, H.H.; Kayun, Z. Comparison of dose calculation algorithms model: Convolution, superposition, and fast superposition in 3-D Conformal Radiotherapy (3D-CRT) treatment plan. J. Phys. Conf. Ser. 2019, 1248, 012047. [CrossRef]

4. Karim, M.K.A.; Sabarudin, A.; Muhammad, N.A.; Ng, K.H. A comparative study of radiation doses between phantom and patients via CT angiography of the intra-/extra-cranial, pulmonary, and abdominal/pelvic arteries. Radiol. Phys. Technol. 2019, 12, 374-381. [CrossRef]

5. Gabriel, C.; Gabriel, S.; Corthout, E. The dielectric properties of biological tissues: I. Literature survey. Phys. Med. Biol. 1996, 41, 2231-2249. [CrossRef] [PubMed]

6. Zell, K.; Sperl, J.I.; Vogel, M.W.; Niessner, R.; Haisch, C. Acoustical properties of selected tissue phantom materials for ultrasound imaging. Phys. Med. Biol. 2007, 52, N475-N484. [CrossRef] [PubMed]

7. In, E. Development of Polymer-Based Gels for Multimodal Medical Imaging Phantoms; TSpace: Toronto, ON, Canada, $2016 ;$ p. 122.

8. Karimi, A.; Shojaei, A. Measurement of the Mechanical Properties of the Human Kidney. IRBM 2017, 38, 292-297. [CrossRef]

9. Hill, D.J.; Preston, C.M.L.; Salisbury, D.J.; Whittaker, A.K. Molecular weight changes and scission and crosslinking in poly(dimethyl siloxane) on gamma radiolysis. Radiat. Phys. Chem. 2001, 62, 11-17. [CrossRef]

10. Bao, C.; Xu, K.-Q.; Tang, C.; Lau, W.-M.; Yin, C.-B.; Zhu, Y.; Mei, J.; Lee, J.; Hui, D.; Nie, H.-Y.; et al. Cross-Linking the Surface of Cured Polydimethylsiloxane via Hyperthemal Hydrogen Projectile Bombardment. ACS Appl. Mater. Interfaces 2015, 7, 8515-8524. [CrossRef]

11. Mazurek, P.; Vudayagiri, S.; Skov, A.L. How to tailor flexible silicone elastomers with mechanical integrity: A tutorial review. Chem. Soc. Rev. 2019, 48, 1448-1464. [CrossRef]

12. Wang, Z.; Volinsky, A.A.; Gallant, N.D. Crosslinking effect on polydimethylsiloxane elastic modulus measured by custom-built compression instrument. J. Appl. Polym. Sci. 2014, 131, 1-4. [CrossRef]

13. McDonald, J.C.; Duffy, D.C.; Anderson, J.R.; Chiu, D.T.; Wu, H.; Schueller, O.J.; Whitesides, G.M. Fabrication of microfluidic systems in poly (dimethylsiloxane). Electrophoresis 2000, 21, 27-40. [CrossRef]

14. Lan, T.; Naguib, H.E.; Coolens, C. Development of a permeable phantom for dynamic contrast enhanced (DCE) imaging quality assurance: Material characterization and testing. Biomed. Phys. Eng. Express 2017, 3, 025018. [CrossRef]

15. Adams, F.; Qiu, T.; Mark, A.; Fritz, B.; Kramer, L.; Schlager, D.; Wetterauer, U.; Miernik, A.; Fischer, P. Soft 3D-Printed Phantom of the Human Kidney with Collecting System. Ann. Biomed. Eng. 2017, 45, 963-972. [CrossRef] [PubMed]

16. Greening, G.J.; Istfan, R.; Higgins, L.M.; Balachandran, K.; Roblyer, D.; Pierce, M.C.; Muldoon, T.J. Characterization of thin poly (dimethylsiloxane)-based tissue-simulating phantoms with tunable reduced scattering and absorption coefficients at visible and near-infrared wavelengths. J. Biomed. Opt. 2014, 19, 115002. [CrossRef]

17. Yu, L.; Skov, A.L. Silicone rubbers for dielectric elastomers with improved dielectric and mechanical properties as a result of substituting silica with titanium dioxide. Int. J. Smart Nano Mater. 2015, 6, 268-289. [CrossRef]

18. Manjunatha, H.C. Comparison of effective atomic numbers of the cancerous and normal kidney tissue. Radiat. Prot. Environ. 2015, 38, 83. [CrossRef]

19. Liu, M.; Sun, J.; Sun, Y.; Bock, C.; Chen, Q. Thickness-dependent mechanical properties of polydimethylsiloxane membranes. J. Micromech. Microeng. 2009, 19. [CrossRef] 
20. Mata, A.; Fleischman, A.J.; Roy, S. Characterization of Polydimethylsiloxane (PDMS) Properties for Biomedical Micro/Nanosystems. Biomed. Microdevices 2005, 2, 281-293. [CrossRef]

21. Ahmad, Z. Polymer Dielectric Materials. Dielectr. Mater. 2012. [CrossRef]

22. Özpolat, Ö.F.; Alım, B.; Şakar, E.; Büyükyıldız, M.; Kurudirek, M. Phy-X/ZeXTRa: A software for robust calculation of effective atomic numbers for photon, electron, proton, alpha particle, and carbon ion interactions. Radiat. Environ. Biophys. 2020, 59, 321-329. [CrossRef] [PubMed]

23. Singh, V.P.; Badiger, N.M.; Kucuk, N. Determination of Effective Atomic Numbers Using Different Methods for Some Low-Z Materials. J. Nucl. Chem. 2014, 2014, 1-7. [CrossRef] 